\title{
Atresia tricúspide: um relato de caso sobre o desafio do diagnóstico intrauterino
}

\author{
Tricuspid atresia: a case report about the challenge of intrauterine diagnosis
}

Atresia tricúspide: reporte de un caso sobre el desafío del diagnóstico intrauterino

Lara Goulart Pereira ${ }^{1 *}$, Anna Tércia de Azevedo Almeida ${ }^{1}$, Fernanda de Paula Vitor ${ }^{1}$, Gabriela Silva Rangel ${ }^{1}$, Lavínia Ribeiro Tavares ${ }^{1}$, Ana Cristina Lopes Albricker ${ }^{1}$.

\section{RESUMO}

Objetivo: Relatar um caso sobre atresia tricúspide (AT), cardiopatia congênita cianogênica rara com elevada mortalidade, evidenciando a importância do diagnóstico intrauterino no planejamento de medidas profiláticas e terapêuticas que permitam o aumento da sobrevida dos pacientes. Detalhamento do caso: Trata-se de paciente portador de AT, atualmente com três anos de idade. Cardiopatia foi suspeitada no período intrauterino por ultrassonografia morfológica e ecocardiografia fetal bidimensional com Doppler em cores e confirmada em ecocardiografia pós-natal. Os neonatos com essa cardiopatia apresentam manifestações clínicas precoces e necessitam de intervenção imediata ao nascimento. O manejo inclui parto cesáreo programado, intervenção precoce com prostaglandina E1 e programação de cirurgias paliativas e corretivas. Atualmente, o paciente encontra-se estável e em boa evolução clínica. Considerações finais: O diagnóstico da AT durante o período fetal possibilita redução da mortalidade e aumento da sobrevida dos portadores devido à intervenção precoce através do parto programado, manejo terapêutico imediatamente após o nascimento e planejamento de correção cirúrgica escalonada.

Palavras-chave: Atresia tricúspide, Diagnóstico precoce, Ecocardiografia Doppler.

\begin{abstract}
Objective: To report a case of tricuspid atresia (TA), a rare cyanogenic congenital heart disease with high mortality, highlighting the importance of intrauterine diagnosis in planning prophylactic and therapeutic measures that allow for increased patient survival. Case details: This is a three years old patient with TA. The heart disease was suspected at intrauterine period by morphological ultrasound and two-dimensional fetal echocardiography color Doppler and confirmed on postnatal echocardiography. Neonates with this heart disease have early clinical manifestations and require immediate intervention at birth. Management included scheduled cesarean delivery, early intervention with prostaglandin E1 and scheduling of palliative and corrective surgeries. Currently, the patient is stable and in good clinical evolution. Final considerations: The diagnosis of TA during the fetal period makes it possible to reduce mortality and increase the survival of patients due to early intervention through scheduled delivery, therapeutic management immediately after birth and planning for staggered surgical correction.
\end{abstract}

Key words: Tricuspid atresia, Early diagnosis, Doppler echocardiography.

\section{RESUMEN}

Objetivo: Reportar un caso de atresia tricuspídea (AT), una cardiopatía congénita cianogénica rara con alta mortalidad, destacando la importancia del diagnóstico intrauterino en la planificación de medidas profilácticas y terapéuticas que permitan aumentar la supervivencia del paciente. Detalles del caso: Se trata de un paciente con AT, actualmente de tres años. Se sospechó cardiopatía en el período intrauterino mediante ecografía morfológica y ecocardiografía fetal bidimensional con Doppler color y se confirmó mediante ecocardiografía posnatal. Los recién nacidos con esta enfermedad cardíaca tienen manifestaciones clínicas tempranas y requieren una intervención inmediata al nacer. El manejo incluyó cesárea programada, intervención temprana con prostaglandina E1 y programación de cirugías paliativas y correctivas. Actualmente el paciente se encuentra estable y con buena evolución clínica. Los recién nacidos con esta enfermedad

${ }^{1}$ Centro Universitário de Belo Horizonte (UNIBH), Belo Horizonte - MG. *E-mail: goulartlara1@gmail.com 
cardíaca tienen manifestaciones clínicas tempranas y requieren una intervención inmediata al nacer. Consideraciones finales: El diagnóstico de AT durante el período fetal permite reducir la mortalidad y aumentar la supervivencia de las pacientes debido a la intervención temprana mediante el parto programado, el manejo terapéutico inmediatamente después del nacimiento y la planificación de la corrección quirúrgica escalonada.

Palabras clave: Atresia tricúspide, Diagnóstico precoz, Ecocardiografía Doppler.

\section{INTRODUÇÃO}

Atresia Tricúspide (AT) é uma cardiopatia congênita cianogênica rara na qual ocorre agenesia ou imperfuração da comunicação atrioventricular direita, o que resulta na ausência de comunicação entre átrio e ventrículo direitos (BRAVO-VALENZUELA NJ, et al., 2018). Essa malformação consiste na quarta principal causa de cardiopatia congênita e cursa com mortalidade de $90 \%$ antes dos dez anos de idade, sendo ligeiramente mais comum no sexo masculino e podendo associar-se a outras malformações cardíacas, como estenose ou atresia pulmonar, transposição de grandes artérias e defeito do septo interatrial ou interventricular. Portadores de AT necessitam de intervenção terapêutica precoce, sendo que o tratamento definitivo é cirúrgico (SUMAL AS, et al., 2020).

A sobrevivência pós-natal do paciente portador de AT depende da possibilidade de direcionamento do sangue venoso do átrio direito para o esquerdo por meio da manutenção do forame oval, da existência de comunicação interatrial e da anastomose entre as circulações sistêmica e pulmonar. Dessa forma, devido à comunicação entre as câmaras atriais direita e esquerda, o sangue não oxigenado proveniente do retorno venoso sistêmico é misturado com o sangue oxigenado transportado pelas veias pulmonares no átrio esquerdo, desencadeando a sobrecarga volumétrica dessa câmera, assim como o aumento do diâmetro do anel da valva mitral e hipertrofia de ventrículo esquerdo que passa a suportar maior fluxo sanguíneo (SUMAL AS, et al., 2020).

Os neonatos portadores dessa cardiopatia geralmente apresentam manifestações clínicas precoces como cianose central, hipoxemia importante e declínio do estado geral durante os primeiros dias de vida. O aumento da sobrevida e o tempo de evolução dos sintomas estão diretamente relacionados ao grau do fluxo pulmonar, sendo que a associação de estenose severa ou atresia pulmonar piora o prognóstico do paciente (MINOCHA PK e PHOON C, 2020). O quadro clínico se agrava progressivamente com o fechamento do forame oval e do canal arterial. Assim, se não houver intervenção terapêutica paliativa imediata, ocorre inviabilidade da circulação do neonato (SUMAL AS, et al., 2020). Essa cardiopatia pode evoluir com complicações como embolia paradoxal, abscesso cerebral, hipoxemia crônica, endocardite e insuficiência cardíaca (ROCHA IEG, et al., 2017).

Devido à gravidade dos sintomas e à rápida deterioração do estado geral dos pacientes portadores de cardiopatias congênitas cianogênicas, é necessário que qualquer anomalia cardíaca potencialmente patológica evidenciada em ultrassonografia fetal seja adequadamente investigada. Nesses casos, a ecocardiografia fetal pode ser empregada. Esse exame permite análise da estrutura e função cardíaca, portanto possibilita o diagnóstico preciso da cardiopatia (DONOFRIO MT, et al., 2014). A impressão diagnóstica obtida no período intrauterino pode ser confirmada no período pós-natal por meio de ecocardiografia transtorácica (BRAVO-VALENZUELA NJ, et al., 2018).

Portanto o diagnóstico da AT durante o período intra-uterino, realizado através do ecocardiograma fetal, é essencial para planejamento de intervenção paliativa no recém-nascido imediatamente após o parto. Tais intervenções visam a manutenção do canal arterial pérvio com o uso de prostaglandinas, preservação da estabilidade hemodinâmica do RN e planejamento de correção cirúrgica escalonada, medidas essenciais para o aumento da sobrevida do paciente (RAO PS, 2019).

Dessa forma, o objetivo desse artigo é evidenciar, mediante o relato de caso, a importância do diagnóstico intrauterino da atresia tricúspide para o planejamento precoce de medidas profiláticas e terapêuticas que permitam o aumento da sobrevida do paciente pediátrico. 


\section{DETALHAMENTO DO CASO}

Trata-se de gestante de 32 anos, primigesta, sem comorbidades e sem intercorrências durante a gestação, sorologias não reagentes, grupo sanguíneo $\mathrm{O}$ e fator $\mathrm{Rh}$ positivo. $\mathrm{Na} 24^{\mathrm{a}}$ semana gestacional realizou ultrassonografia morfológica na qual houve suspeição de malformação cardíaca fetal, sendo indicada ecocardiografia fetal com Doppler. Este exame foi realizado na 35a semana de gestação e demonstrou situs solitus em levocardia, forame oval pérvio com fluxo da direita para esquerda, conexão atrioventricular concordante, valva tricúspide atrésica, septo interventricular exibindo descontinuidade de cerca de 3 milímetros com fluxo bidirecional, ventrículo direito hipoplásico, valva pulmonar atrésica e artéria pulmonar com calibre levemente reduzido com fluxo retrógrado (Figura 1).

Figura 1 - Ecocardiografia fetal com Doppler em cores realizada na 35쪼 semana gestacional.

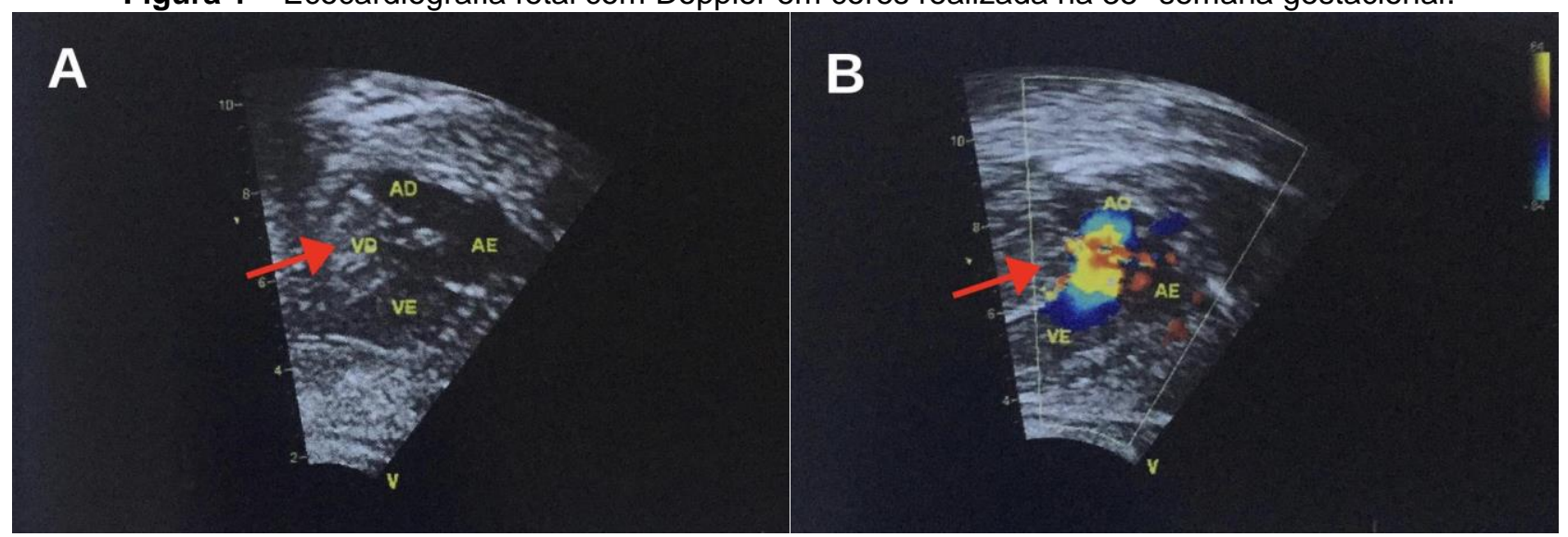

Legenda: A: Ventrículo direito hipoplásico em ecocardiografia fetal bidimensional com Doppler; B: Ausência de fluxo sanguíneo em ventrículo direito em ecocardiografia fetal bidimensional com Doppler em cores. $\mathrm{Na}$ imagem: AD: Átrio direito, AE: Átrio esquerdo, VD: Ventrículo direito, VE: Ventrículo esquerdo.

Fonte: Pereira LG, et al., 2021.

Os achados patológicos na ecocardiografia fetal e a idade gestacional em que foi realizada, contribuíram para a indicação de parto cesáreo programado, ao completar 38 semanas gestacionais, devido à necessidade de intervenção pós natal imediata. Não houve intercorrências durante o parto e o neonato do sexo masculino nasceu com peso adequado para idade gestacional, em boas condições, índice de Apgar de primeiro minuto 6 pontos e de quinto minuto 9 pontos, sem alterações ao exame físico sumário.

Foi realizada intervenção com infusão contínua de prostaglandina E1 no período perinatal e internação em unidade de terapia intensiva. Evoluiu com apneia secundária ao uso deste medicamento, com necessidade de intubação orotraqueal. Realizada ecocardiografia transtorácica com Doppler em cores que confirmou o diagnóstico de atresia das valvas tricúspide e pulmonar.

Após 14 dias de vida, o paciente foi submetido ao procedimento Blalock-Taussig modificado, evoluindo com descompensação hemodinâmica no pós-operatório imediato, sendo medicado com adrenalina por 72 horas e ácido acetilsalicílico $5 \mathrm{mg} / \mathrm{Kg}$ ao dia. Evoluiu com insuficiência cardíaca congestiva e foi iniciado uso de furosemida $1 \mathrm{~g} / \mathrm{Kg} / \mathrm{dia}$. Devido a complicações apresentadas em pós operatório, neonato permaneceu internado em Unidade de Terapia Intensiva por 25 dias e em enfermaria por 6 dias, recebendo alta hospitalar com 45 dias de vida.

Aos cinco meses de idade foi realizado cateterismo para implantação de stent para manutenção do fluxo sanguíneo adequado, no qual não obteve o resultado esperado. Submetido ao procedimento de BlalockHanlon por toracotomia transesternal mediana. Ao completar 12 meses de vida, o lactente realizou procedimento de Glenn bidirecional, sem intercorrências intra-operatórias. Atualmente paciente encontra-se com três anos de idade, estável e em boa evolução clínica, em uso diário de ácido acetilsalicílico, sem episódios de descompensação hemodinâmica desde a última intervenção cirúrgica. A previsão futura é a realização do procedimento de Fontan aos quatro anos de idade. 
O relato de caso apresentado neste artigo possui autorização de publicação assinado pela responsável legal do paciente, sob forma de Termo de Consentimento Livre e Esclarecido. O documento encontra-se disponível, caso seja necessária comprovação ética.

\section{DISCUSSÃO}

Atresia Tricúspide (AT) é uma malformação cardíaca rara com alta taxa de mortalidade se não houver intervenção precoce (SUMAL AS, et al., 2020). Cardiopatias congênitas estão presentes em 0,2 a $4 \%$ das gestações no ocidente e são as malformações congênitas associadas à maior morbimortalidade (ROSA RCM, et al., 2013; LAGE EM e BARBOSA A, 2012). Entretanto, a taxa de mortalidade associada às malformações cardíacas está reduzindo devido aos avanços das técnicas cirúrgicas (LOPES SAVA, et al., 2018). O diagnóstico da AT durante o período fetal possibilita redução das taxas de mortalidade e aumento da sobrevida dos portadores dessa cardiopatia, uma vez que permite realização de parto planejado e intervenção profissional desde o nascimento (BRAVO-VALENZUELA NJ, et al., 2018).

Para rastreamento de malformações estruturais cardíacas, assim como de anomalias cromossômicas e outros defeitos estruturais, pode ser realizada medida da translucência nucal (TN) por ultrassonografia de primeiro trimestre gestacional, idealmente entre 11 e 14 semanas (RAMOS BA, et al., 2017). O aumento da TN é o principal parâmetro sugestivo de malformação cardíaca estrutural na análise ultrassonográfica obstétrica, portanto presença de TN aumentada constitui indicação para investigação complementar (HERNANDEZ-ANDRADE E, et al., 2017).

Análise ultrassonográfica obstétrica é útil na detecção de grande quantidade de malformações estruturais com identificação de até $90 \%$ das cardiopatias congênitas no período pré-natal. Esse exame permite visualização das câmaras cardíacas e vias de saída, portanto em portadores de AT pode haver ventrículo direito hipoplásico, dilatação do ventrículo esquerdo e valva tricúspide ecogênica, impérvia e imóvel, coexistência de comunicação interventricular e transposição de grandes vasos. Deve ser avaliado o tamanho do forame oval, sendo que sua redução está associada com hidropsia e sofrimento fetal (BRAVOVALENZUELA NJ, et al., 2018).

A American Heart Association (AHA) recomenda que a investigação de cardiopatias congênitas por ecocardiografia fetal seja realizada na população de alto risco, o que inclui gestantes portadoras de doença cardíaca estrutural, outros filhos nascidos com cardiopatias congênitas, achado suspeito em ultrassonografia morfológica ou aumento da translucência nucal acima do percentil 95. Ressalta-se que a gestante mencionada neste relato não apresentava fatores de risco gestacionais e a TN em ultrassonografia de primeiro trimestre encontrava-se dentro dos valores de normalidade. Entretanto, foram visualizadas anomalias estruturais na ultrassonografia morfológica realizada em $24^{a}$ semana gestacional, o que representa um risco absoluto maior que $40 \%$ de doença cardíaca congênita e é uma clara indicação para ecocardiografia fetal (DONOFRIO MT, et al., 2014).

Ecocardiografia fetal é o exame padrão-ouro para diagnóstico de cardiopatias congênitas no período intrauterino e possui maior sensibilidade que o ultrassom obstétrico (TAGUE L, et al., 2017). Detecção de cardiopatia congênita pela ecocardiografia fetal, quando suspeitada por anormalidade cardíaca visualizada por ultrassom obstétrico em gestantes de baixo risco, é maior que $40 \%$. Esse exame possibilita a análise da anatomia e biometria cardiovascular, além do estudo do fluxo sanguíneo por Doppler, portanto permite diagnóstico adequado de cardiopatias congênitas (DONOFRIO MT, et al., 2014).

Assim, ecocardiografia fetal deve ser realizada preferencialmente entre 18 e 22 semanas gestacionais, mas apresenta resultados aceitáveis desde o fim do primeiro trimestre até o termo (DONOFRIO MT, et al., 2014). A ecocardiografia transabdominal a partir da $13^{\text {a }}$ semana permite adequada análise da anatomia cardíaca, sendo que se realizada antes desse período, deve ser realizado por via transvaginal (MORPHY SS, et al., 2020). A gestante mencionada no caso realizou ecocardiografia na $35^{\mathrm{a}}$ semana gestacional devido à dificuldade em encontrar profissionais com expertise suficiente para a realização do exame com qualidade necessária para o diagnóstico correto. No entanto, ainda assim, foi possível o diagnóstico intrauterino de AT. 
A conduta terapêutica depende do grau de fluxo sanguíneo pulmonar, que pode estar aumentado ou reduzido de acordo com a existência de defeitos cardíacos associados. Pacientes com hiperfluxo possuem shunt esquerda-direita devido à comunicação interventricular e ausência de estenose ou agenesia pulmonar associada, ou presença de transposição de grandes artérias (SUMAL AS, et al., 2020). Geralmente cursam com cianose leve ou ausente nas primeiras horas após nascimento, sendo que manifestações clínicas mais evidentes, como insuficiência cardíaca, ocorrem tardiamente quando há queda da resistência vascular pulmonar (ATIK E, et al., 2019).

Pacientes com hipofluxo pulmonar, como o recém-nascido relatado neste artigo, apresentam restrição da via de saída do fluxo sanguíneo em direção às artérias pulmonares, ou seja, há comunicação interventricular ausente ou restritiva associada ou não à estenose ou agenesia pulmonar. Dessa forma, o fechamento fisiológico do canal arterial nesses pacientes implica na impossibilidade ou restrição grave da passagem do sangue da circulação venosa sistêmica para a circulação pulmonar, clinicamente percebido como piora do quadro de cianose e rápida deterioração clínica (SUMAL AS, et al., 2020).

Assim, intervenção precoce no período perinatal tem como objetivo manutenção do shunt sistêmicopulmonar para possibilitar oxigenação sanguínea até realização de correção cirúrgica definitiva. Em outras palavras, casos de AT que dependem exclusivamente do canal arterial para oxigenação sanguínea devem sofrer intervenções com objetivo de manter o shunt entre as circulações sistêmica e pulmonar. Se não forem instituídas, a circulação do neonato torna-se incompatível com a vida (SUMAL AS, et al., 2020). Portanto a conduta pós-natal imediata deve ser administração contínua intravenosa de prostaglandina E1 (PGE1) para manutenção do canal arterial aberto. O uso de PGE1 é uma conduta paliativa e não consiste no tratamento permanente da AT (RAO PS, 2019).

Outra intervenção profilática que visa a manutenção do shunt sistêmico-pulmonar é o procedimento de Blalock-Taussig modificado. Essa correção cirúrgica consiste na interposição entre as artérias subclávia e pulmonar com uso de enxerto sintético, por meio de toracotomia mediana, com objetivo de manter o paciente hemodinamicamente estável e sem restrições ao exercício até que haja possibilidade de correção cirúrgica definitiva (RAO PS, 2019; KIRAN U, et al., 2017). O paciente relatado apresentava restrição grave do fluxo pulmonar devido à comunicação interventricular restritiva e estenose pulmonar, sendo necessária propedêutica para manutenção do fluxo e boa oxigenação sanguínea. Assim, foi realizada intervenção profilática com infusão de PGE1 no período perinatal e optado por cirurgia de Blalock-Taussig modificada no $14^{\circ}$ dia de vida para controle hemodinâmico até que seja realizado tratamento definitivo.

Outro fator importante para preservar um quadro hemodinâmico viável é a manutenção do shunt direitaesquerda por meio do forame oval pérvio, uma vez que a ausência de comunicação interatrial nesses pacientes significa que não há via de saída do átrio direito (SUMAL AS, et al., 2020). Assim, em casos de obstrução interatrial por fechamento do forame oval, há necessidade de correção cirúrgica (RAO PS, 2019). Pode ser realizado procedimento de Blalock-Hanlon, que consiste na abertura de orifício no septo interatrial que permite a comunicação das cavidades atriais direita e esquerda com consequente melhora da oxigenação sanguínea (PENHA JG, et al., 2015).

No entanto, sabe-se que o tratamento definitivo da AT ocorre através de correção cirúrgica escalonada (FROCK BW, et al., 2017). Inicialmente, é realizado o procedimento de Glenn em crianças maiores de três meses, no qual é confeccionado shunt cavopulmonar no qual a veia cava superior é anastomosada à artéria pulmonar. Assim, o sangue venoso da circulação sistêmica é direcionado para um ou ambos os pulmões (sendo denominado uni ou bidirecional, respectivamente) e não mais para o átrio direito. Esse procedimento é o estágio inicial da correção cirúrgica (SHARMA R, 2018).

Posteriormente é realizada a operação de Fontan, segunda etapa cirúrgica, em média um ano após correção de Glenn. Esse procedimento consiste na criação de desvio do fluxo da veia cava inferior para artéria pulmonar, com oclusão da junção cavoatrial, formando shunt cavopulmonar total (CORNO AF, et al., 2019). É importante ressaltar que os procedimentos de Glenn e Fontan não visam a correção da AT, mas representam uma alternativa para tornar a circulação compatível com a vida (RAO PS, 2019). Estudos 
realizados no período pós-natal em pacientes portadores de AT submetidos ao tratamento cirúrgico demonstraram taxa de sobrevida entre 81 a $89 \%$ no primeiro ano de idade (TURCIOS KIP e MADRID WKC, 2018).

Foi optado, para o paciente mencionado neste artigo, realização do procedimento de Blalock-Hanlon com 5 meses de idade para manutenção do shunt direita-esquerda e aos 12 meses, procedimento de Glenn bidirecional com confecção de shunt sistêmico-pulmonar. É importante ressaltar que o paciente se manteve estável hemodinamicamente desde o último procedimento cirúrgico até os dias atuais. A literatura analisada corrobora com o manejo clínico e cirúrgico deste caso e comprova que sua instituição precoce contribuiu para o aumento da sobrevida do paciente.

Sendo assim, sabe-se que a AT é uma cardiopatia congênita cianogênica rara, com alto índice de mortalidade neonatal e incompatível com a vida caso não haja intervenções terapêuticas precoces (SUMAL AS, et al., 2020). O método diagnóstico para identificação dessa patologia é ecocardiografia fetal, que deve ser realizada em fetos com aumento da translucência nucal ou outra alteração sugestiva em ultrassonografia obstétrica e na presença de fatores de risco para malformações cardíacas (DONOFRIO MT, et al., 2014). Sabe-se que o diagnóstico precoce intrauterino da AT possibilita aumento da sobrevida dos pacientes portadores dessa cardiopatia devido à possibilidade de intervenção precoce através do parto programado, manejo terapêutico imediatamente após o nascimento e planejamento de correção cirúrgica escalonada (BRAVO-VALENZUELA NJ, et al., 2018).

\section{REFERÊNCIAS}

1. ATIK E, et al. Caso 3/2019-Atresia Tricúspide IIB, em Evolução Natural, com 21 Anos de Idade. Arquivos Brasileiros de Cardiologia, 2019; 112: 592-593.

2. BRAVO-VALENZUELA NJ, et al. Prenatal diagnosis of congenital heart disease: A review of current knowledge. Indian heart journal, 2018; 70(1): 150-164.

3. CORNO AF, et al. Physiological Fontan Procedure. Frontiers in pediatrics, 2019; 7: 196.

4. DONOFRIO MT, et al. Diagnosis and treatment of fetal cardiac disease: a scientific statement from the American Heart Association. Circulation, 2014; 129(21): 2183-2242.

5. FROCK BW, et al. Living with Tricuspid Atresia: Case Report with Review of Literature. Neonatal Network, 2017; 36(4): 218-228.

6. HERNANDEZ-ANDRADE E, et al. Early evaluation of the fetal heart. Fetal diagnosis and therapy, 2017; 42(3): 161173.

7. KIRAN U, et al. The blalock and taussig shunt revisited. Annals of cardiac anaesthesia, 2017; 20(3): 323-330.

8. LAGE EM, BARBOSA AS. Cardiopatias e gravidez. Femina, 2012; 43(1): 44-50.

9. LOPES SAVA, et al. Mortalidade para Cardiopatias Congênitas e Fatores de Risco Associados em Recém-Nascidos, Um Estudo de Coorte. Arquivos Brasileiros de Cardiologia, 2018; 111(5): 666-673.

10. MINOCHA PK, PHOON C. Tricuspid atresia. StatPearls [Internet], 2020.

11. MORHY SS, et al. Posicionamento sobre Indicações da Ecocardiografia em Cardiologia Fetal, Pediátrica e Cardiopatias Congênitas do Adulto. Arquivos Brasileiros de Cardiologia, 2020; 115: 987-1005.

12. PENHA JG, et al. Senning paliativo no tratamento de cardiopatias congênitas com hipertensāo pulmonar grave. Arquivos Brasileiros de Cardiologia, 2015; 105(4): 353-361.

13. RAMOS BA, et al. Valor preditivo da translucência nucal para cromossomopatias, 2017; 74175(23).

14. RAO PS. Management of Congenital Heart Disease: State of the Art-Part II-Cyanotic Heart Defects. Children, 2019; 6(4): 54.

15. ROCHA IEG, et al. Atresia Tricúspide e Dupla Via de Saída do Ventrículo Esquerdo: Uma Rara Associação em Vida Adulta. ABC., imagem cardiovasc, 2017; 30(1): 31-35.

16. ROSA RCM, et al. Cardiopatias congênitas e malformações extracardíacas. Revista Paulista de Pediatria, 2013; 31(2): 243-251.

17. SHARMA R. The bidirectional Glenn shunt for univentricular hearts. Indian Journal of Thoracic and Cardiovascular Surgery, 2018; 34(4): 453-456.

18. SUMAL AS, et al. Tricuspid atresia: Where are we now?. Journal of Cardiac Surgery, 2020; 35(7): 1609-1617.

19. TAGUE L, et al. Common Findings in Late-Gestation Fetal Echocardiography. Journal of Ultrasound in Medicine, 2017; 36(12): 2431-2437.

20. TURCIOS KIP, MADRID WKC. Diagnóstico prental de atresia tricuspidea. Revista de la Federación Centroamericana de Obstetricia y Ginecología, 2018; 2016(21). 